\title{
Instrumentos Baseados em Psicologia Positiva no Brasil: uma Revisão Sistemática
}

\author{
Jeferson Gervasio Pires - Universidade Federal de Santa Catarina, Florianópolis, Brasil \\ Maiana Farias Oliveira Nunes - Universidade São Francisco, Itatiba, Brasil \\ Carlos Henrique Sancineto da Silva Nunes - Universidade Federal de Santa Catarina, Florianópolis, Brasil
}

\begin{abstract}
Resumo
A Psicologia Positiva estuda fatores que potencializam o desenvolvimento de aspectos positivos nos seres humanos, nos grupos e nas instituições. Com o objetivo de mapear instrumentos para avaliação de fenômenos propostos pela Psicologia Positiva, efetuou-se uma revisão sistemática de 49 publicações nacionais levantadas em todos os índices de busca do BVS-Psi. Observou-se um aumento na produção sobre avaliação em Psicologia Positiva, envolvendo o estudo de diversificados construtos, destacando-se qualidade de vida, resiliência, coping e bem-estar, além de estudos sobre a construção e adaptação de instrumentos para avaliá-los. Foram identificados 34 construtos e 67 instrumentos, apesar de haver prevalência de estudos concentrados em oito construtos e onze instrumentos.

Palavras-chave: Psicologia Positiva, testes psicológicos, avaliação psicológica.
\end{abstract}

\section{Brazilian Instruments Based on Positive Psychology: A Systematic Review}

\begin{abstract}
Positive Psychology refers to the study of the development of positive aspects of human beings, groups and institutions. Having the aim of knowing instruments based on Positive Psychology, we performed a systematic review referring to 49 national publications from all search indexes of BVS-Psi. An increase in Positive Psychology production was observed, involving the study of diverse constructs such as life quality, coping and resilience and well-being, as well as studies on the construction and adaptation of instruments to assessing them. 34 constructs and 67 instruments were identified, though there was a prevalence of studies focused on eight constructs and eleven instruments.

Keywords: Positive Psychology, psychological tests, psychological assessment.
\end{abstract}

Instrumentos Brasileños Basados en Psicología Positiva: Revisión sistemática

\begin{abstract}
Resumen
La Psicología Positiva estudia factores que potencializan el desarrollo de aspectos positivos en los seres humanos, en los grupos y en las instituciones. Con el objetivo de rastrear instrumentos para la evaluación de fenómenos propuestos por la Psicología Positiva, se realizó una revisión sistemática de 49 publicaciones nacionales, derivadas de todos los índices de búsqueda de la BVS-Psi. Se observó un aumento en la producción sobre evaluación en Psicología Positiva, lo que implica el estudio de diversos constructos, como por ejemplo, calidad de vida, resiliencia, coping y bienestar, además de estudios sobre la construcción y adaptación de instrumentos para evaluarlos. Se identificaron 34 constructos y 67 instrumentos, pese a haber prevalencia de estudios concentrados en ocho constructos y 11 instrumentos.

Palabras clave: Psicología Positiva, tests psicológicos, evaluación psicológica.
\end{abstract}

\section{Introdução}

A Psicologia Positiva é a área que propõe o estudo científico das forças e virtudes próprias do indivíduo, que faz com que os psicólogos adotem uma postura mais apreciativa em relação ao potencial, motivação e capacidades humanas (Sheldon \& King, 2001). Mais recentemente, Niemiec (2010) afirmou que a Psicologia Positiva promove o estudo científico do que há de melhor e mais forte nos indivíduos, nas famílias e na sociedade. Frequentemente, descreve-se que o movimento da Psicologia Positiva teve início em meados da década de 1990, ocasião em que Martin Seligman presidia a APA (Associação Americana de Psicologia), sugerindo à classe psicológica uma mudança no foco das pesquisas e das intervenções (Scorsolini-Comin \& Santos, 2010), visando incluir aspectos positivos, em detrimento da exclusividade dada aos aspectos desadaptados, patológicos e negativos, focos da psicologia tradicional.

O campo da Psicologia Positiva tem como meta ajudar as pessoas para que atinjam um ótimo nível de funcionamento (Macdonald, Bore, \& Munro, 2008), ou ainda, como propõe Peterson (2006), estuda o que torna a vida digna de ser bem vivida. Dentre os construtos trabalhados na Psicologia Positiva, pode-se citar como exemplo qualidade de vida, esperança, bem-estar subjetivo, autoeficácia e outros. Pode-se considerar que os focos residem no estudo das emoções positivas, nas características individuais positivas e nas instituições 
positivas (desde famílias a locais de trabalho, que dão ênfase ao potencial humano) (Park \& Peterson, 2006; Seligman, 2004).

No que se refere à produção nacional em Psicologia Positiva, não se sabe exatamente qual teria sido o primeiro estudo publicado, porém, Pacico e Bastianello (2014) e Paludo e Koller (2007) concordam que tenha sido um estudo com autoria de Hutz, Koller e Bandeira (1996), sobre resiliência. As autoras indicam que, desde então, houve crescimento na produção em Psicologia Positiva nacionalmente, ressaltando, inclusive, estudos envolvendo construtos, como esperança, autoeficácia, autoestima, otimismo e afetos.

Uma revisão integrativa sobre os instrumentos de avaliação relacionados à Psicologia Positiva no contexto brasileiro (entre 1970 e 2008) foi efetuada por Scorsolini-Comin e Santos (2010) e resgatou 246 estudos nas bases Lilacs e Scielo. Contudo, em observância aos critérios de exclusão, restaram apenas seis itens para a análise, os quais envolviam publicações entre 2004 e 2007. Os autores relatam a pouca quantidade dos trabalhos que operacionalizam os construtos estudados pela Psicologia Positiva no Brasil, apesar de inferirem crescimento no futuro e, além disso, de enfatizarem que os fenômenos mais frequentemente abordados referiam-se ao bem-estar subjetivo e à satisfação geral.

Ainda em relação ao artigo, outra importante informação refere-se ao fato de os instrumentos mais utilizados serem originalmente estrangeiros, havendo apenas um desenvolvido em âmbito nacional (Escala de Bem-Estar Subjetivo - EBES), sendo que os demais possuíam apenas tradução e adaptação semântica ao português brasileiro. Adicionalmente, os autores relatam que o pico de produção aconteceu entre 2006 e 2007 e informam que a população idosa foi a mais frequentemente abordada nesses estudos.

Em uma revisão sistemática a respeito da produção científica em Psicologia Positiva no Brasil, elaborada por Pureza, Corazza, Castro e Lisboa (2012), foram analisados 19 de 39 documentos resgatados nas bases BVS - Psi, Scielo e BDTD (Biblioteca brasileira de teses e dissertações), até o ano de 2012. As autoras enfatizaram que o interesse científico associado à Psicologia Positiva esteja acontecendo de forma incipiente no Brasil, conforme sugerido por Paludo e Koller (2007). O pico de produção descrito foi entre 2007 e 2008, sendo que os principais construtos abordados referiam-se ao bem-estar, satisfação em domínios específicos, flow, emoções positivas, saúde e autoestima. Quanto às áreas em que os estudos foram conduzidos, as autoras destacaram psicologia escolar, relações familiares, processos básicos e psicologia da saúde. Com relação aos instrumentos mais utilizados, a EBES recebeu destaque pela maior frequência de uso. No que tange ao perfil das amostras envolvidas nas pesquisas, a faixa etária adulta foi a mais frequente, tendo havido apenas um estudo com público infantil e adolescente, sendo que as principais características dos participantes foram casais, seguida por universitários, professores e alunos. Por fim, os estudos de correlação foram os mais frequentes nos documentos analisados.

Em observância à realidade apresentada, do estágio ainda incipiente apontado pelas pesquisas de levantamento dos trabalhos envolvendo avaliações de fenômenos abordados pela Psicologia Positiva no Brasil, cabe pesquisar a atual produção de instrumentos voltados para ela no país. Com esse objetivo, revisou-se, sistematicamente, a literatura científica brasileira a fim de levantar informações que permitam observar a evolução da área no cenário nacional. Essa revisão poderá representar uma contribuição para a área por apresentar sua atual produção, cuja informação permitirá analisar o desenvolvimento referente aos instrumentos baseados em fenômenos estudados em Psicologia Positiva no Brasil, apontando as lacunas e também as áreas que têm sido consistentemente abordadas.

\section{Método}

\section{Material e Procedimentos}

Foram incluídos trabalhos científicos completos e exclusivamente brasileiros, como artigos, trabalhos de conclusão de curso (TCCs), teses e dissertações, indexados por meio do BVS-Psi (www.bvs-psi.org.br). Definiu-se, como critérios de inclusão, que o estudo abordasse construção, adaptação, validação ou aplicação de algum instrumento relacionado à Psicologia Positiva. Os acessos às bases de dados ocorreram nos dias $10 \mathrm{e}$ 11 de julho de 2014 e foram utilizados os descritores Instrumento Psicologia Positiva, Instrumentos Psicologia Positiva e Escala Psicologia Positiva. A partir de tais procedimentos, foram resgatados 360 resultados. Para a seleção dos estudos que seriam analisados nesta revisão, os critérios de exclusão adotados foram: a) tema central da publicação não ser relacionado à Psicologia Positiva, b) estudos em repetição em outra base, c) estudos descritos de forma incompleta (resumos), d) estudos originários de outros países, e) estudos sem a utilização de algum instrumento relacionado à Psicologia Positiva e, ainda, f) as revisões da literatura. Em observância aos critérios de exclusão, restaram 49 estudos. 
Para o levantamento e a organização dos dados, construiu-se um protocolo com 14 categorias de análise. Essas categorias são descritas a seguir.

Ano de publicação: refere-se ao ano da publicação, descrito no material.

Região geográfica: região brasileira na qual a universidade do primeiro autor está localizada, sendo elas, norte, sul, nordeste, sudeste e centro-oeste. Quando o artigo foi publicado em coautoria, a região dos demais autores não foi considerada.

Categoria da Instituição de Ensino Superior IES: tipo de IES à qual o primeiro autor está vinculado na publicação, podendo ser pública ou privada.

Área de conhecimento do autor: refere-se à área de formação do primeiro autor. Essa informação foi obtida a partir da consulta ao currículo lattes do primeiro autor, resgatando sua formação na graduação.

Fenômeno Abordado: refere-se aos fenômenos abordados nos estudos, no âmbito da Psicologia Positiva.

Contexto de Aplicação: refere-se ao contexto ou local no qual o trabalho foi desenvolvido, podendo ser empresa, escola, clínica, hospital, universidade ou no contexto do esporte.

Instrumentos Utilizados: aborda os instrumentos usados no estudo, como, por exemplo, EBES, escala de Satisfação com a Vida e PANAS.

Quantidade de Participantes: referente ao número de participantes descrito no documento analisado.

Idade dos Participantes: refere-se a faixa etária dos participantes da pesquisa, sendo categorizados nos grupos de crianças (zero a 11 anos), adolescentes (12 a 18 anos), adultos (19 a 59 anos), idosos (a partir de 60 anos) ou população mista (mais de uma faixa etária).

Modalidade dos Participantes: relaciona-se ao perfil dos participantes descritos na pesquisa, como alunos de escolas ou de universidade, pacientes, colaboradores de empresas, professores, profissionais da saúde, dentre outros.

Material de Coleta de Dados: tipo de instrumento utilizado para a coleta dos dados, podendo ser um instrumento em lápis e papel ou online, entrevista ou misto (mais de um tipo de procedimento).

Tipos de Estudo: aborda o objetivo do estudo, coletado a partir de verbos utilizados na descrição do objetivo do texto, podendo ser validação, correlação, adaptação e construção.

Revista de Publicação: revista científica na qual a pesquisa foi publicada.

Qualis da Revista: indica a avaliação Qualis da revista na qual o material foi publicado, de acordo com o conceito apresentado no ano de 2014. Essa informação foi obtida por meio da consulta ao site qualiscapes. gov.br

No que se refere à organização dos dados, considerando que, em alguns artigos, as variáveis Instrumentos e Fenômenos abordados apresentavam número variado, decidiu-se considerar o número máximo de três itens, sendo os demais omitidos da análise do presente artigo. Especificamente em relação à variável Fenômenos abordados, descartou-se também aqueles que não eram temas alvos da Psicologia Positiva, como violência e crenças parentais. Os fenômenos resiliência e coping foram agrupados e, no que se refere à variável tipos de estudo, aqueles que apresentavam o verbo "explorar" no objetivo foram considerados correlação.

Os dados levantados foram analisados com base na frequência de informações referentes às 14 categorias listadas previamente, a partir do software estatístico Stata 12. A descrição dos resultados contemplou mais amplamente os aspectos que obtiveram frequência acima de dois e, em razão disso, em duas variáveis não foram apresentados os casos que obtiveram frequência única. $\mathrm{Na}$ variável Instrumentos, por exemplo, levantou-se o total de 67, porém, foram apresentados e discutidos apenas onze deles. O mesmo ocorreu com a variável Fenômenos, que revelou 34 fenômenos distintos, dos quais foram apresentados e discutidos apenas oito. Por fim, em relação à variável área de conhecimento do autor, foram consideradas mais de uma formação de graduação, quando assim descrito no lattes.

\section{Resultados}

Em relação ao ano das publicações analisadas, elas foram publicadas entre 2000 e 2014, período no qual se pôde observar um aumento dessas produções, especialmente, no que se refere ao período compreendido entre 2008 e 2013. A respeito da faixa que vai de 2000 até 2007, exclusivamente em 2003, resgatou-se o total de três documentos, contudo, nos demais anos, a quantidade de publicações permaneceu estável e baixa, com uma publicação por ano. Houve um pico de publicações em $2008(f=9)$, conforme detalhado na Figura 1.

No que se refere à região brasileira de onde se originaram as publicações utilizadas nesta revisão $(f=$ 48), destacaram-se as regiões sudeste, com maior quantidade de publicações $(f=26)$ e a região sul $(f=18)$. $\mathrm{Na}$ sequência, apareceram as regiões centro oeste e nordeste, obtendo a mesma frequência $(f=2)$ cada uma. 


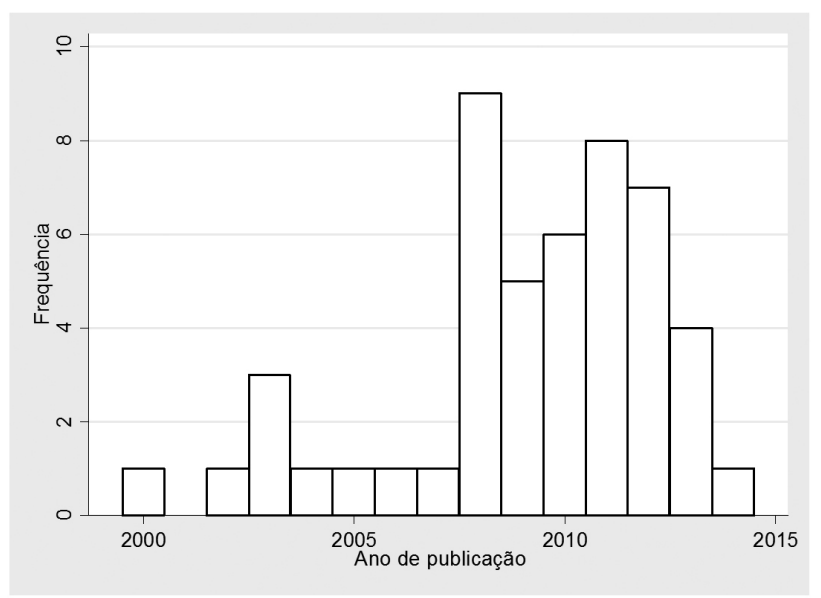

Figura 1. Quantidade de publicações por ano.

Sobre essa variável, cabe salientar que um documento não informava a Universidade do primeiro autor. Associado a essa variável estão as instituições de ensino $(f=$ 46), sobre as quais houve predominância de produções derivadas de instituições públicas $(f=28)$ em comparação com as privadas $(f=18)$. Em três produções analisadas, não estavam informadas as IES de vinculação do primeiro autor.

Em relação à faixa etária dos participantes $(f=46)$, observou-se que a maioria das publicações referia-se a sujeitos adultos $(f=22)$, seguido da faixa de idade mista $(f=10)$. Houve também estudos com idosos $(f=7)$, adolescentes $(f=4)$ e, por último, a população infantil $(f=3)$. Três documentos analisados não informaram a faixa etária dos participantes. Em consideração à quantidade de participantes das pesquisas, observou-se uma variação entre 16 e 1.064 sujeitos, conforme detalhado na Tabela 1.

Quanto ao perfil dos participantes, 11 estudos envolveram alunos de escolas de ensino fundamental e médio, mesma frequência apresentada pelos estudantes universitários, seguida por pacientes $(f=7)$. Houve a participação de colaboradores de empresas e casais, representando cinco artigos cada um, além dos profissionais da saúde e dos idosos, com dois artigos para cada grupo recém-mencionado.

A respeito das áreas de conhecimento dos autores $(f=47)$, destaca-se que 37 delas referiam-se à Psicologia, seguido pela Enfermagem e Medicina, representado quatro ocorrências cada uma delas e, por fim, duas publicações eram referentes a área de Educação Física. Em dois casos, contudo, não foi possível localizar o
Tabela 1

Quantidade de Participantes (N)

\begin{tabular}{lcc}
\hline $\begin{array}{l}\text { Quantidade de } \\
\text { participantes }\end{array}$ & Frequência & Percentual \\
\hline 1 a 100 & 10 & 20 \\
101 a 300 & 22 & 42 \\
301 a 500 & 9 & 20 \\
501 a 700 & 5 & 10 \\
701 a 1000 & 2 & 5 \\
1001 a 1064 & 1 & 3 \\
\hline Total & 49 & 100 \\
\hline
\end{tabular}

currículo lattes do primeiro autor, a partir do nome descrito na publicação.

Com relação ao contexto nos quais estes estudos foram realizados $(f=42)$, houve predominância do escolar $(f=22$, considerando os níveis de ensino fundamental até o universitário). O contexto empresarial apresentou sete ocorrências, ao passo que hospitalar e domiciliar obtiveram a frequência de cinco cada um. Por fim, o contexto clínico apresentou a menor frequência $(f=3)$. Destaca-se que em sete documentos analisados não foi especificado o contexto de aplicação ou de coleta de dados.

Foram identificados 34 fenômenos distintos nas pesquisas consultadas, contudo, houve predominância de estudos relacionados a oito construtos, quais sejam, qualidade de vida, resiliência e coping, bem-estar subjetivo, satisfação com a vida, autoeficácia, criatividade e bem-estar espiritual. Essa informação é detalhada na Tabela 2.

Em relação aos instrumentos utilizados, identificou-se uma variedade de 67 deles, destacando-se, pela maior frequência, a escala de Autoestima de Rosenberg, o WHOQOL - Bref, a escala de Bem-Estar Subjetivo - EBES, a escala de Satisfação Conjugal, a escala de Bem-Estar Espiritual, a escala de Satisfação de Vida para Crianças (Giacomoni \& Hutz) e a escala de satisfação no trabalho, conforme Tabela 3.

No que tange aos materiais usados para a coleta dos dados $(f=48)$, houve predominância de instrumentos no formato de lápis e papel $(f=38)$, seguido pelo formato misto, o qual se compõe por instrumento escrito e entrevista $(f=10)$. Destaca-se que um artigo 
consultado não informou essa variável. Com relação à variável tipo de estudo $(f=49)$, destaca-se que houve predominância dos correlacionais $(f=37)$, os quais buscavam, sobretudo, correlações entre aspectos positivos ou entre esses aspectos e dados sociodemográficos, seguidos pelos estudos de validação $(f=6)$, adaptação $(f$ $=3$ ) e de construção de instrumentos $(f=3)$.

Em relação às revistas nas quais estes documentos estão publicados $(f=43$, sendo os outros seis teses ou dissertações), cabe salientar a variedade dos resultados, com as publicações ocorrendo em 25 periódicos distintos. Destacou-se a revista Psico-USF, com cinco publicações, seguida pelas revistas Aletheia, Arquivos Brasileiros de Psicologia, Estudos de Psicologia

Tabela 2

Relação de Fenômenos com Maiores Frequências

Fenômenos abordados Frequência Percentual

\begin{tabular}{lcc}
\hline Qualidade de vida & 9 & 21 \\
Resiliência e Coping & 9 & 21 \\
Bem-estar subjetivo (BES) & 7 & 15 \\
Autoeficácia & 6 & 13 \\
Afetos & 5 & 11 \\
Satisfação com a vida & 4 & 9 \\
Criatividade & 2 & 5 \\
Bem-estar espiritual & 2 & 5 \\
\hline Total & 44 & 100 \\
\hline
\end{tabular}

(Campinas), Psicologia Reflexão e Crítica, revista Associação Médica Brasileira, revista Psicologia Educacional e Escolar e Revista da Educação Física, as quais apresentaram a frequência de dois artigos cada. As demais revistas tiveram apenas 1 (um) artigo publicado, não sendo mencionadas por esse motivo.

Quanto à avaliação Qualis das revistas $(f=43)$, foi possível constatar que 19 artigos estavam publicados em revistas com conceito A2, procedidas pelo conceito $\mathrm{B} 1(f=8)$. As revistas com conceitos A1 e B2 representam cinco ocorrências cada uma, seguida pelas revistas com conceitos B3 $(f=4)$ e B4 $(f=2)$. Cabe lembrar que as teses e dissertações não puderam ser analisadas por não possuírem tal avaliação.

\section{Discussão}

Os resultados apontaram para algumas questões já conhecidas em Psicologia, como, por exemplo, certo desequilíbrio na produção científica em Psicologia Positiva quanto às regiões do país, destacando-se a sudeste, seguida pela região sul (Costa et al., 2012). A produção dessas regiões, em conjunto, representou mais de $89 \%$ de toda a produção sobre Psicologia Positiva do país, sendo considerada bastante expressiva.

As universidades públicas mantiveram a liderança em termos de quantidade de publicações na área. Contudo, destaca-se a representatividade da produção derivada das universidades privadas nestas pesquisas, contribuindo substancialmente com as produções nacionais sobre Psicologia Positiva.

Tabela 3

Instrumentos Descritos com Frequência Maior que Um

\begin{tabular}{lcc}
\hline Instrumentos utilizados & Frequência & Percentual \\
\hline Escala de Autoestima de Rosenberg (adaptado) & 6 & 20 \\
WHOQOL - Bref. (adaptado) & 3 & 10 \\
EBES (nacional) & 3 & 10 \\
Escala de Satisfação Conjugal (adaptado) & 3 & 10 \\
Escala do Bem-Estar Espiritual (adaptado) & 3 & 10 \\
Escala de Satisfação no Trabalho (nacional) & 3 & 10 \\
Dyadic Adjustment scale (DAS) (adaptado) & 2 & 6 \\
Escala de Satisfação de Vida Infantil (Giacomoni \& Hutz) (nacional) & 2 & 6 \\
Escala de Satisfação com a Vida (Chaves) (nacional) & 2 & 6 \\
Escala de Enfrentamento (COPE) (adaptado) & 2 & 6 \\
PANAS (adaptado) & 2 & 6 \\
\hline Total & 31 & 100 \\
\hline
\end{tabular}


No que tange ao crescimento da produção na área, tanto de instrumentos utilizados, quanto de construtos abordados nas pesquisas sobre Psicologia Positiva, os achados estão em consonância com Pacico e Bastianello (2014), contudo, divergem das revisões de Pureza et al. (2012) e de Scorsolini-Comin e Santos (2010). Em relação ao fato, houve prevalência de estudos concentrados em instrumentos e construtos específicos, em detrimento de uma variedade mais ampla. Como exemplo, o número de escalas para avaliar, especificamente, o construto satisfação com a vida foi muito alto, enquanto outros fenômenos só apresentaram uma escala para sua avaliação. Essa concentração pode ser um indicador positivo, uma vez que com isso aprofunda-se o conhecimento sobre tais temáticas. Esse dado mostra também coerência com o que ocorreu na área nos Estados Unidos, em que os primeiros estudos voltavam-se para o que torna as pessoas felizes (operacionalizando-se as pesquisas com instrumentos voltados para bem-estar, satisfação com a vida, afetos, etc.) e, posteriormente, ampliando os focos de estudo. Contudo, destaca-se a importância em se estudar mais ampla e profundamente a diversidade de construtos em Psicologia Positiva. Esse achado corrobora a reflexão proposta por Scorsolini-Comin e Santos (2010).

Ainda em relação aos instrumentos, esta revisão observou que há uma diversidade deles, sobretudo em relação aos adaptados para uso no Brasil, o que converge com a descrição de crescimento de produção, efetuada por Pacico e Bastianello (2014). Houve frequente utilização do instrumento EBES, observando-se esse dado tanto nesta revisão, quanto no trabalho desenvolvido por Pureza et al. (2012). Com relação aos demais instrumentos, porém, o mesmo não ocorreu. Sobre isso, destaca-se, por exemplo, que a escala de Autoestima de Rosenberg e o WHOQOL - Bref apareceram em destaque nesta revisão, contudo, não foram citados em outros trabalhos consultados (Pureza et al, 2012; Scorsolini-Comin \& Santos, 2010). Essa informação pode ser um indicativo do aumento da diversidade da produção, conforme descrito por alguns autores.

No que se refere à produção em Psicologia Positiva de forma geral, foi possível identificar um aumento na construção e adaptação de instrumentos estrangeiros. É possível destacar uma evidência de aumento de produção, especialmente, se comparadas à quantidade de pesquisas resgatadas presentemente com as revisões de Scorsolini-Comin e Santos (2010) e de Pureza et al. (2012).

No que tange aos principais fenômenos abordados, foi observada uma diversidade de construtos.
Houve prevalência dos estudos envolvendo qualidade de vida, resiliência e coping, bem-estar subjetivo e afetos, fato que discorda dos achados de Pureza et al. (2012), contudo, na revisão de Scorsolini-Comin e Santos (2010), bem-estar subjetivo foi descrito como um dos mais frequentes, relacionando-se com o observado nesta revisão.

O resultado dessa comparação parece apontar para outro indicativo de aumento da variabilidade nos temas da produção científica, uma vez que parte dos construtos observados na corrente revisão parece ser nova, quando comparados com os descritos nas demais. Nesse aspecto, o único construto que foi convergente nas três revisões foi satisfação, contudo, ele apareceu com destaque nas duas revisões, e frequência intermediária na corrente. De forma inversa, destaca-se que os construtos resiliência e coping, e criatividade não foram mencionados nas outras revisões, apesar de terem aparecido com alguma frequência nesta.

No que se refere às áreas de conhecimento mais frequentes de onde derivam as pesquisas de Psicologia Positiva, houve maior frequência de publicações da Psicologia, como era de se esperar. Contudo, constatou-se considerável participação de profissionais de outras áreas da saúde, como Enfermagem, Medicina e Educação Física, especialmente em relação aos construtos qualidade de vida e satisfação, fato que confirma a natureza multiprofissional de alguns construtos, conforme sugerido por Keyes, Shmotkin e Ryff (2002) e por Reppold, Serafini e Menda (2014). Nessa mesma direção, é possível comentar a respeito da diversidade de contextos e de revistas nos quais as pesquisas aconteceram e foram publicadas, outra evidência em relação à natureza multiprofissional de alguns focos de estudo da Psicologia Positiva.

Em relação à modalidade mais frequente de participantes, destacaram-se os alunos de escolas de ensino fundamental e médio, seguidos pelos universitários. A grande ocorrência das pesquisas no contexto educacional é uma prática comum nas diversas áreas de pesquisa em Psicologia, o que pode se relacionar tanto com o interesse em estudar esses fenômenos nessa etapa do desenvolvimento como pela facilidade de acesso a grande número de participantes em poucas idas a campo. $\mathrm{O}$ interesse pela população escolar parece fazer sentido com a noção de educação positiva proposta por Seligman (2011), para quem as emoções têm papéis fundamentais no aprendizado. Nesse sentido, há um forte movimento internacional no qual pesquisas em larga escala estão sendo realizadas para verificar o papel de 
competências socioemocionais como potencializadores do desenvolvimento no contexto educacional (Santos \& Primi, 2014). Salienta-se que uma parte significativa das competências socioemocionais são construtos propostos a partir do referencial da Psicologia Positiva. Tais pesquisas são de extrema relevância, pois apresentam propostas práticas de intervenções que catalisem o desenvolvimento de competências socioemocionais em crianças e adolescentes.

Sobre a variável faixa etária, a população idosa foi uma das menos frequentes, cujo fato denota pouca atenção da Psicologia Positiva em relação à referida população. Essa realidade pode ter alguma coincidência com a produção em Psicologia, de forma geral, o que deve ser investigado em outros estudos.

No que tange aos formatos mais utilizados nos instrumentos para a coleta de dados, observou-se que as escalas e inventários (categorizados aqui como "instrumentos") foi o meio mais frequente. Esse dado encontra-se em consonância com as observações de Diener, Lucas e Oishi (2005) e Layous e Zanon (2014), em relação ao uso de instrumentos de autorrelato em Psicologia Positiva. Vale destacar que, com o crescente uso de ferramentas de coleta de dados online, há uma tendência de mudança desse resultado, conforme descrevem Wachelke, Natividade, De Andrade, Wolder e Camargo (2014). Contudo, em nenhum dos estudos levantados encontrou-se esse tipo de coleta.

Dentre os tipos de estudos mais frequentes, foi possível destacar os correlacionais. A esse respeito, ressalta-se que os aspectos positivos têm sido comparados especialmente com dados sociodemográficos, tal como iniciado por Wilson (1967) há aproximadamente cinco décadas, convergindo também com os resultados da revisão de Pureza et al., (2012). Ademais, é importante destacar que os estudos de construção e adaptação apresentaram maior frequência. Isso reflete e corrobora a viés empírico da Psicologia Positiva, descrito por Seligman (2011), além de indicar um movimento de amadurecimento conceitual na área, com a ocorrência de pesquisas que discutem a dimensionalidade dos instrumentos e a eficácia das medidas geradas por estes. Por fim, ressalta-se que as revistas nas quais estes estudos foram publicados são variadas e que a avaliação Qualis é excelente (a maioria das revistas possuía avaliação "A" no Qualis).

Em que pese o fato da Psicologia Positiva ser conhecida como o campo de estudo científico do que há de melhor e mais forte nos indivíduos, nas famílias e na sociedade (Niemiec, 2010), com a meta a auxiliar pessoas na obtenção de um ótimo nível de funcionamento (Macdonald et al., 2008), parece ser necessário maior investimento por parte dos pesquisadores do campo, especialmente, no que diz respeito à abrangência dos fenômenos estudados e à publicação de instrumentos de avaliação diversificados. Acredita-se que isso poderá contribuir com o avanço da Psicologia Positiva no Brasil.

\section{Considerações Finais}

O presente estudo destinou-se a realizar uma revisão sistemática de 49 publicações nacionais indexadas pelo BVS-Psi, com o intuito de investigar os instrumentos que avaliam construtos estudados pela Psicologia Positiva no Brasil. Observou-se que o país está passando por uma fase de crescimento em relação à produção científica em Psicologia Positiva, especialmente no que se refere à construção, adaptação e validação de instrumentos. Quanto aos fenômenos mais estudados, verificou-se maior investimento em pesquisas sobre qualidade de vida e bem-estar, juntamente com Coping e resiliência. Isso aponta, de certa forma, um atraso do Brasil na pesquisa em Psicologia Positiva, uma vez que, em outros países, observa-se maior variabilidade dos fenômenos avaliados na atualidade. Qualidade de vida e bem-estar foram as temáticas que deram início às pesquisas da área na década de 1990 e, ainda no período atual, observa-se maior concentração das pesquisas brasileiras nessas temáticas. Assim, identifica-se a necessidade de ampliação dos focos de estudo e de construção de instrumentos, de modo a testar as hipóteses teóricas elaboradas na área na realidade sóciocultural no país e, também, contribuir para o avanço do conhecimento científico nesse campo, indo além do que já se sabe sobre os fenômenos psicológicos considerados positivos.

Uma limitação deste estudo refere-se ao número de dados levantados em oito variáveis analisadas (região, instituições de ensino, faixa etária dos participantes, área de conhecimento dos autores, contexto de aplicação, material de coleta de dados, revistas e conceito Qualis), as quais apresentaram número inferior de ocorrências por falta de informação disponível nos documentos consultados. Por fim, sugere-se que novas pesquisas abordem mais amplamente a diversidade de construtos possíveis em Psicologia Positiva, especialmente nos instrumentos de avaliação psicológica brasileiros e, também, que seja verificada a eficácia deles para monitorar intervenções psicológicas com essa abordagem. 


\section{Referências}

Costa, J. P., Costa, A. L. F., Lima, F. C., Seixas, P. S., Pessanha, V. C., \& Yamamoto, O. H (2012). A produção científica sobre a formação de psicólogos no Brasil. Psicologia em Pesquisa, 6(02), 130-138. doi: 10.5327/Z1982-12472012000200006

Diener, E., Lucas, R. E., \& Oishi, S. (2005). Subjective well-being: The science of happiness and life satisfaction. In C. R. Snyder \& S. J. Lopez (Eds.), Handbook of positive psychology (2a ed.), (pp. 63-73). New York, NY: Oxford University Press. Retirado de https://internal.psychology.illinois.edu/ reprints/index.php?site_id $=24$

Giacomoni, C. H, \& Hutz, C. S. (2008). Escala multidimensional de satisfação de vida para crianças: Estudos de construção e validação. Estudos de Psicologia Campinas, 25(1), 23-35. Recuperado de http:// www.scielo.br/pdf/estpsi/v25n1/a03v25n1.pdf

Hutz, C. S., Koller, S. H., \& Bandeira, D. R. (1996). Resiliência e vulnerabilidade em crianças em situação de risco. Coletâneas da Anpepp, 1(12), 75-90.

Keyes, C. L. M., Shmotkin, D., \& Ryff, C. D. (2002). Optimizing well-being: The empirical encounter of two traditions. Journal of Personality and Social Psychology, 82(6), 1007-1022. Retirado de http://www. midus.wisc.edu/findings/pdfs/62.pdf

Layous, K., \& Zanon, C. (2014). In Claudio. S. Hutz (Org.). Avaliação em psicologia positiva. Porto Alegre: Artmed. pp 23-42.

Macdonald, C., Bore, M., \& Munro, D. (2008). Values in action scale and the Big 5: An empirical indication of structure. Journal of Research in Personality, 42, 787-799. doi: 10.1016/j.jrp.2007.10.003.

Niemiec, R. M. (2010). Character strengths and positive psychology: On the horizon in family therapy. The Family Psychologist, 26(1), 16-17. doi:10.1080/17439 760.2014 .888581

Pacico, J., Bastianello, C., \& Micheline R. (2014). In Claudio. S. Hutz (Org.). Avaliação em psicologia positiva. Porto Alegre: Artmed. pp 101-110.

Paludo, S. S., \& Koller, S. H. (2007). Psicologia positiva: Uma nova abordagem para antigas questões. Paidéia. 17(36), 9-20. Retirado de http://www.scielo. br/pdf/paideia/v17n36/v17n36a02.pdf
Park, N., \& Peterson, C. (2006). Moral competence and character strengths among adolescents: The development and validation of the Values in Action Inventory of Strengths for Youth, Journal of Adolescence, 29(6) 891-909. doi:10.1016/j. adolescence.2006.04.011

Peterson, C. (2006). A primer in positive psychology. New York: Oxford University Press.

Pureza, J. R., Corazza, C. H. K., Castro, E. K., \& Lisboa, C. S. M. (2012). Psicologia positiva no Brasil: uma revisão sistemática da literatura. Revista Brasileira de Terapia Cognitiva Vol. 8 no 2 - Jul./Dez. Retirado de http://www.rbtc.org.br/detalhe_artigo. asp?id $=168$

Reppold, C. T., Serafini, A. J., \& Menda, S. C. (2014). In Claudio. S. Hutz. (Org.). Avaliação em Psicologia Positiva. Porto Alegre: Artmed. pp 121-146.

Santos, D., \& Primi, R. (2014). Desenvolvimento socioemocional e aprendizado escolar: Uma proposta de mensuração para apoiarpolíticas públicas. São Paulo: Instituto Ayrton Senna.

Seligman, M. E. P. (2011). Florescer: Uma nova compreensão sobre a natureza da felicidade e do bem- estar. Trad. Cristina Paixão Lopes. Rio de Janeiro: Objetiva.

Scorsolini-Comin, F., \& Santos, M. A.. (2010). Psicologia positiva e os instrumentos de avaliação no contexto brasileiro. Psicologia: Reflexão e Crítica, 23(3), 440-448. doi: 10.1590/S0102-79722010000300004

Sheldon, K. M., \& King, M. (2001). Why positive psychology is necessary? American Psychologist, 36(3), 216-217. Retirado de http://web.missouri. edu/ sheldonk/pdfarticles/AP01.pdf

Wachelke, J., Natividade, J., De Andrade, A., Wolter, R., \& Camargo, B. (2014). Caracterização e avaliação de um procedimento de coleta de dados on-line com recrutamento presencial (CORP). Avaliação Psicológica, 13, 143-146. Retirado de http://www. redalyc.org/pdf/3350/335030683017.pdf

Wilson, W. (1967). Correlates of avowed hapiness. Psychological Bulletin, 67, 294-306.

Recebido: 05/12/2014

$1^{a}$ reformulação: 19/01/2015

Aprovado: 23/02/2015 
Sobre os autores:

Jeferson Gervasio Pires é graduado em Psicologia pelo Centro Universitário Estácio de Sá de Santa Catarina e mestrando em Psicologia, na linha de Avaliação em Saúde e Desenvolvimento, na Universidade Federal de Santa Catarina (UFSC).

Maiana Farias Oliveira Nunes é graduada em Psicologia pela Faculdade Ruy Barbosa, possui mestrado e doutorado em Psicologia pela Universidade São Francisco, atualmente é pesquisadora colaboradora da USF, UFRGS e UFSC, e atua na Convergente Consultoria, com planejamento de pesquisas e análises psicométricas.

Carlos Henrique Sancineto da Silva Nunes é graduado, mestre e doutor em Psicologia pela Universidade Federal do Rio Grande do Sul, professor adjunto da UFSC e tem atuado na área de Avaliação Psicológica, com ênfase em avaliação da personalidade, inteligência, habilidades socioemocionais e psicometria.

\section{Contato com os autores:}

Universidade Federal de Santa Catarina

Centro de Filosofia e Ciências Humanas, Departamento de Psicologia

Trindade - 88040-970 - Florianópolis - SC, Brasil

E-mail: jefersongp@gmail.com 